\title{
Erratum: GV/m Single-Cycle Terahertz Fields from a Laser-Driven Large-Size Partitioned Organic Crystal [Phys. Rev. Lett. 112, 213901 (2014)]
}

\author{
C. Vicario, B. Monoszlai, and C. P. Hauri
}

(Received 8 November 2018; published 17 December 2018)

DOI: 10.1103/PhysRevLett.121.259901

For the electric field strength $E_{p}$ calculation, a prefactor was omitted. For the estimation of the peak THz field generated in the PCS crystal, the experimental pulse energy, the focus size, and the pulse envelope duration $\tau_{\mathrm{FWHM}}$ is taken into account. The electric field can be expressed as

$$
E_{p}=\sqrt{\frac{\Sigma_{\text {pulse }}}{c \epsilon_{0} A \tau_{\mathrm{FWHM}}}},
$$

with $\Sigma_{\text {pulse }}$ the pulse energy measured with a calibrated Golay cell detector taking into account the losses. THz pulse energies up to $12 \mu \mathrm{J}$ were measured by illuminating the PCS crystal with a $3 \mathrm{~mm}$ pump diameter beam. $A$ is the effective area for a two-dimensional Gaussian distribution with a FWHM measured in the experiment of $270 \mu \mathrm{m}$ : $A \approx \mathrm{FWHM}^{2} \pi /[4 \ln (2)] . c$ and $\epsilon_{0}$ are the speed of light and the vacuum permittivity, respectively. The half-maximum THz pulse duration $\tau_{\text {FWHM }}$ is derived from the Gaussian fit of the envelope of $|E(t)|^{2}$ of the electro-optical sampling shape reported in Fig. 2(a) and is equal to 364 fs. According to Fig. 1, the THz peak field is about $0.38 \mathrm{GV} / \mathrm{m}(3.8 \mathrm{MV} / \mathrm{cm})$, and the corresponding magnetic field about $1.3 \mathrm{~T}$. The corrected electro-optical field evolution is reported in Fig. 1.

The effective electric and magnetic peak field by pumping the entire PCS crystal surface would result in about $2.6 \mathrm{GV} / \mathrm{m}$ and $8.6 \mathrm{~T}$, respectively (instead of $3.6 \mathrm{GV} / \mathrm{m}$ and $12 \mathrm{~T}$ ).

Furthermore, there is a typo in the reported frequency range (p. 3). It correctly reads, "The experimental focus was reproduced in ZEMAX simulation modeling the THz radiation as discrete frequencies between $0.5 \mathrm{and} 6 \mathrm{THz}$ [inset of Figs. 3(a) and 3(b)]." We would like to thank J. Faist and H. Schift for having pointed out the mistake in the field calculation.

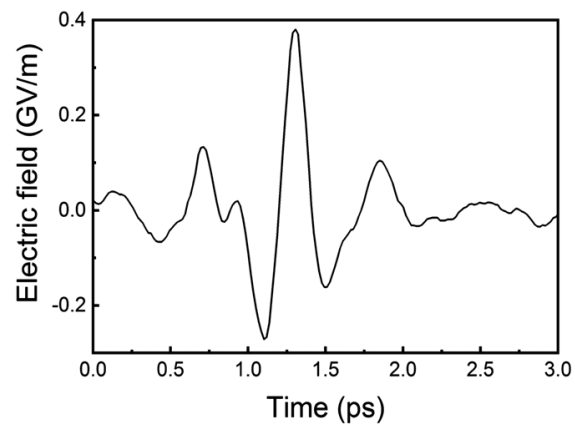

FIG. 1. THz field measured with electro-optical sampling and rescaled using Eq. (E1). 